\title{
CUIDADO DE ENFERMERÍA Y SATISFACCIÓN DEL ADULTO MAYOR, HOSPITAL SANTA MARIA DEL SOCORRO DE ICA-2014
}

\author{
Susana Alvarado Alfaro ${ }^{1,2, a, b}$ \\ ${ }^{1}$ Facultad de Enfermería de la Universidad Nacional San Luis Gonzaga de Ica
}

Nursing care and satisfaction of elderly Hospital Santa Maria Socorro Ica-2014

${ }^{2}$ Hospital Regional de Ica.

a Licenciada en Enfermería, ${ }^{\circ}$ Doctor en Enfermería.

\section{RESUMEN}

Objetivo: Analizar la relación del cuidado de enfermería, con la satisfacción del adulto mayor, en el Hospital Santa María del Socorro de Ica, durante septiembre y octubre del 2014. Materiales y Métodos: Estudio, descriptivo, correlacional, transversal, con 80 adultos mayores de 65 a 80 años de edad, hospitalizados en los servicios de medicina y cirugía. Resultados: En forma global, se encontró un nivel de cuidado humanizado bueno (53,7\%), en menor proporción fue excelente (46,3\%), así mismo $68,7 \%$ se mostraron satisfechos, $21,3 \%$ poco satisfechos, y $10 \%$ muy satisfechos, con el cuidado de enfermería recibido. El cuidado humanizado se relaciona con la satisfacción por el servicio de enfermería recibido $\left(X^{2}=11,0 \mathrm{p}, 000\right)$. En cuanto a las categorías, el hacer de la enfermería $\left(X^{2}=26,9\right)$, el apoyo emocional $\left(X^{2}=14,1\right)$, y la empatía $\left(X^{2}=11,0\right)$, se relacionan con la satisfacción por el servicio de enfermería. Conclusiones: Los adultos mayores hospitalizados, manifestaron excelente y buena percepción del cuidado humanizado brindado por las enfermeras, especialmente en las categorías: Proactividad, apoyo emocional, apoyo físico, sentimientos del paciente, y priorización para ser cuidado.

Palabras clave: Cuidado, enfermería, satisfacción, adultos mayores hospitalizados.

\section{SUMMARY}

Objective: To analyze the relationship of nursing care and the satisfaction of the elderly, at the Santa Maria Hospital Relief Ica, during September and October 2014. Materials and Methods: A descriptive, correlational, cross, with 80 adults aged 65-80 years old, hospitalized in medicine and surgery. Results: Overall, the level of human care good $(53,7 \%)$ was found to a lesser extent was excellent (46,3\%), also $68,7 \%$ were satisfied, $21,3 \%$ dissatisfied and $10 \%$ very satisfied with the nursing care received. The humanized care is related to the satisfaction with nursing service received $(X 2=11,0 \mathrm{p}, 000)$. As for classes, make nursing ( $X 2=26,9)$, emotional support $(X 2=14,1)$, and empathy $(X 2=11,0)$, are related to the satisfaction of the nursing service. Conclusions: Older adults hospitalized, expressed excellent and good perception of the humanized care provided by nurses, especially in the categories: Proactive, emotional support, physical support, feelings of the patient, and prioritization to be careful.

Keywords: Care, nursing, satisfaction, elderly hospitalized

\section{INTRODUCCIÒN}

Los avances tecnológicos, médicos, clínicos y procedimentales, plantean en la actualidad en los servicios de salud, que los profesionales tengan conocimientos especializados; en el campo de la enfermería estos además deben ir acompañados de calidez, y de cuidados humanizados. En el día a día las enfermeras comprobamos que las instituciones hospitalarias están impregnadas del modelo asistencial y curativo, reduciendo lo humano a lo biológico, alejando el ejercicio profesional de las enfermeras de su visión humanística y holística. Al respecto Watson (1) señala que el cuidado humanizado se apoya en el conocimiento científico, la capacidad técnica y la relación terapéutica que el enfermero establece con el paciente, y supone acoger al otro de una manera cálida sin dejar de ser uno mismo, despojándose de todos los factores externos que en algún momento puedan afectar la atención comprometida y de óptima calidad que implica el cuidado humanizado. 
Alvarado $S$.

Según Healt Canadá para el año 2025, 15\% o más de la población superará los 60 años de edad. (2). Este envejecimiento poblacional es un fenómeno multidimensional con consecuencias económicas, sociales, políticas y para el sector salud representa todo un desafío que es necesario empezar a resolverlo. A pesar de que la prolongación de la vida humana es un éxito de la sociedad moderna, surgen problemáticas de salud nunca antes vistas, tales como el incremento de las enfermedades crónicas y degenerativas, de la dependencia y de la transformación de las características de los usuarios de los servicios de salud (3). Estos cambios sugieren que el tema del envejecimiento de la población ocupe un lugar prioritario en la agenda de los gobiernos, los sistemas de salud y la educación, donde todavía se percibe que no existe una respuesta adecuada al problema.

En el Perú, según el Instituto Nacional de Estadística e Informática INEI-Censo 2007, existían 2'495,866 de personas adultas mayores, que representaba el $9.1 \%$ de la población nacional y en el año 2025 será el $13,27 \%$. En Ica, según la misma fuente en el censo del 2007 se registró 68,746adultos mayores de 60 años, y la esperanza de vida que en el año 2000 fue de 73,6 años, para el 2020 será de 78,2 años (4).

Para la ejecución de la presente investigación se han identificado estudios importantes respecto del cuidado de enfermería y la satisfacción del adulto mayor, entre los cuales destacan los de: Romero-Massa, Contreras $M$, Pérez $P$, Moncada A, y Jiménez $Z$ (5), quienes encontraron excelente y buena percepción del cuidado humanizado brindado por las enfermeras, en las Categorías: Priorizar el Cuidado, Dar Apoyo Emocional, Características de la Enfermera y sus Cualidades del Hacer; y Disponibilidad para la Atención; Rojas Martínez, y Barajas Lizarazo (6), hallaron que el nivel de percepción de los usuarios, respecto de la calidad de cuidado de enfermería, es de mediana importancia; Tejada Cruz, K. (7), señala cierta relación entre los factores sociodemográficos con el tiempo de hospitalización, y con la calidad de atención del cuidado de enfermería hacia el adulto mayor; López Amasifén W, Rodríguez Curi K. (8), también muestran altos porcentajes de satisfacción del Adulto Mayor respecto de los cuidados que brinda la enfermera en el servicio de Medicina; Molina Cardona (9), que encontró $83 \%$ de alto grado de satisfacción con la calidad del cuidado de enfermería; Corrales M. (10), señala un nivel alto para el $52 \%$ de adultos mayores, sobre el cuidado recibido; González Burgos, y Quintero Martínez (11), quienes mencionan que el 73\% de las mujeres hospitalizadas, siempre percibieron el cuidado humanizado del personal de enfermería; Cabarcas $\mathrm{O}$, Montes C, Ortiz G. (12), que encontraron $85 \%$ de buena satisfacción del paciente hospitalizado con la atención de enfermería, 15\% regular; y Peroza Krigmar, Querakes Francy, Silva Maira (13), quienes dan a conocer sobre el Cuidado Humanizado, y encuentran $100 \%$ de pacientes afirmando que la enfermera los trata con respeto, amabilidad e igualdad, $85 \%$ que la enfermera respeta su autonomía, $85 \%$ que se satisfacen sus necesidades de atención, 95\% que la enfermera le explica los procedimientos que realiza, $\quad 95 \%$ coincidieron que la enfermera le dedica el tiempo necesario para atenderlo, y que les brinda atención las 24 horas del día.

Históricamente, la acción de cuidar siempre ha sido dinámica, y ha trascendido según el enfoque teórico con que se abordó (Antropológica, Sociológica, Cultural, Religiosa, Psicológica, Económica, Medica, etc), el cuidar va asociado a múltiples factores y es multidimensional, está relacionado directa $\mathrm{e}$ indirectamente con quien da y quien recibe el cuidado. En las investigaciones que se mencionan se puede corroborar tal dinámica, en ellas ha quedado demostrado que la percepción de la calidad del cuidado de enfermería se encuentra relacionada con la satisfacción de los usuarios.

Comprender los fundamentos ontológicos del cuidado y sus componentes teóricos permiten, dar un sentido coherente y consistente a la enfermería como una ciencia humana. Por ello 
la teoría de Jean Watson es útil, permite plantear una filosofía de cuidados, un lenguaje teórico propio y una relación entre teoríapráctica que revitaliza aspectos no visibles de la enfermería como los que plantea Sarter (14) en su revisión de las fuentes filosóficas de la teoría de Watson: El alma (espíritu, o interior de sí), el dualismo (experiencias subjetivas/ objetivas), la armonía, la casualidad y el tiempo, la evolución espiritual, y la autotrascendencia, aspectos que han estado siempre presentes en enfermería, que han sido opacados o subvalorados por el enfoque positivista y tecnológico. La teoría de Jean Watson, de corte existencialista, humanista y con una profunda influencia de la Teoría de Levinás es un ejemplo que da cuenta de la necesidad de rescatar los elementos básicos del cuidado, que permitirán su trascendencia.

Según Watson (15), el cuidado debe ser profesional, estructurado, formalizado y destinado a satisfacer las necesidades del ser humano con el propósito de promover, mantener o recuperar la salud. En el ámbito de enfermería, se ha publicado diversa y variada literatura, sobre filosofía, teorías y modelos; quizás porque la disciplina está tomando más fuerza y visibilidad en el tema de los cuidados y su gestión, ejes centrales de los discursos actuales de enfermería. El cuidado es una actividad que concierne a todos los profesionales de la salud, sin embargo enfermería, como profesión sanitaria, es la que destina más esfuerzo, tiempo y dedicación a los cuidados.

El cuidado tiene dos dimensiones: una inmersa en la cultura, con sus prácticas individuales y colectivas; y la otra, la moral, que asigna al cuidado un valor, un bien, que legitima el actuar. Es así que las teorías de enfermería que se han desarrollado en las últimas décadas han vislumbrado un desarrollo teórico hacia los cuidados, como lo señala la literatura anglosajona en que destacan los pensamientos de Rogers (16), Leininger (17), Parse (18), Pender (19), Newman (20), entre otros.

La investigación "Cuidado de Enfermería, y Satisfacción del Adulto Mayor, en el Hospital
Santa María del Socorro de Ica-2014", analiza la relación del cuidado de enfermería, con la satisfacción del adulto mayor; sabiendo que las enfermeras tienen como misión cuidar la salud de los pacientes, y que sus intervenciones están basadas en principios científicos, humanísticos y éticos, con respeto a la vida y a la dignidad humana

\section{MATERIAL Y MÉTODOS.}

Investigación no experimental, cuantitativa, descriptiva - correlacional, transversal, que analiza la relación del cuidado de enfermería, con la satisfacción del adulto mayor, en el Hospital Santa María del Socorro de Ica, durante los meses de septiembre y octubre del 2014. La muestra fue intencional, constituida por 80 adultos mayores de 65 a 80 años de edad, hospitalizados en los servicios: Medicina 66 pacientes y Cirugía 14 pacientes.

Se utilizó el cuestionario "Percepción de comportamientos de cuidado humanizado de enfermería" (PCHE), de Rivera y Triana (21), basado en la teoría de Watson, que consta de 50 preguntas en escala de Likert de 4 puntos, en donde 1 representa a nunca, 2 algunas veces, 3 casi siempre y 4 siempre, estructurado en nueve categorías: Cuidado de Enfermería, Sentimientos del paciente, Características de la enfermera, Apoyo emocional, Apoyo físico, Cualidades del hacer de la enfermera, Proactividad, Empatía, Priorizar al ser de cuidado, y Disponibilidad para la atención.

Las técnicas aplicadas fueron: La encuesta, la observación, el análisis y la síntesis. La información para llevar a cabo la investigación fue recogida de fuentes directas e indirectas, siendo:

a) Fuentes directas: Entrevistas a los adultos mayores.

b) Fuentes indirectas: Análisis documental, de libros, textos, investigaciones, tesis, Internet, y otros.

El análisis se facilitó con estadísticos descriptivos: frecuencias, porcentajes, media aritmética, desviación estándar, mediante el estadístico SPSS V 21,0 Se correlaciono el Cuidado de Enfermería y sus 9 dimensiones con la Satisfacción, mediante el estadístico Ji 
Cuadrada, para 95\% de confianza. También se correlaciono el cuidado global con los datos sociodemográficos, mediante asociación multivariada, y bivariada de Pearson.

\section{RESULTADOS.}

En la Tabla 1 se muestra que, $53,7 \%$ de los Adultos Mayores hospitalizados percibieron que el Cuidado Humanizado de Enfermería fue bueno, y $46,3 \%$ que fue excelente. Este resultado estaría evidenciando que los servicios de medicina y cirugía del Hospital santa María del Socorro de Ica, cuentan con personal de enfermería que brinda buena atención, al Adulto Mayor.

\section{Tabla 1. Percepción del cuidado humanizado de enfermería}

\begin{tabular}{|c|c|}
\hline Percepción global & $\%$ \\
\hline Excelente & $46,3 \%$ \\
\hline Bueno & $53,7 \%$ \\
\hline Total & $100 \%$ \\
\hline
\end{tabular}

En la tabla 2, se muestra $43,7 \%$ de buenos y $38,7 \%$ de excelentes sentimientos de las enfermeras hacia los Adultos Mayores; que grafica la interacción entre la enfermera y el paciente.

En cuanto a las características de la Enfermera, se evidencia una percepción de $65 \%$ de buenas y $7,5 \%$ de excelentes características, tales como la empatía que tuvo la enfermera con el adulto mayor, amabilidad en el trato, eficiencia en el desempeño de sus tareas, claridad al comunicarse, estímulos positivos, y cuidado comprometido y solidario.

En la categoría del Hacer de la Enfermera, $76,2 \%$ de los adultos mayores, percibieron buena, y 2,5\% excelente, el desempeño de la enfermera; se valoraron el conocimiento y habilidades profesionales, liderazgo en el equipo de trabajo, trabajo en equipo, facilidad en el diálogo, explicación anticipada de los procedimientos, y acciones de excelencia en el cuidado.

En la categoría Proactividad, 60\% valoraron como excelente, y $26,2 \%$ buena; la interacción de la enfermera con el adulto mayor antes de realizar los cuidados, así como la respuesta de las enfermeras a sus preguntas, las instrucciones sobre su cuidado, la ayuda con la información para que pueda tomar decisiones, y la confianza para que llame si tiene problemas.

En la categoría Apoyo Emocional de la enfermera, $58,7 \%$ de los adultos mayores, percibieron como excelente, y 27,5\% bueno; dicho apoyo consistió en la inspiración de confianza, la compañía durante los procedimientos médicos, la tranquilidad que transmitían las enfermeras, y el cuidado amoroso, y dedicado, durante su estadía en el servicio.

En la categoría Apoyo Físico recibido, 51,3\% percibieron excelente, y $32,4 \%$ bueno, es decir cuando la enfermera hacia que el paciente se sienta bien físicamente, lo miraba cuando le hablaban, le ayudaban a disminuir el dolor físico, atendían sus necesidades básicas (baño, eliminación, alimentación), y se esforzaban por brindar comodidad física.

En la categoría Empatía, se encontró 73,7\% de buena y 3,8\% excelente empatía. es decir la relación cercana de la enfermera con el adulto mayor, a quienes expresaban sus sentimientos sobre la enfermedad y el tratamiento, establecían una relación agradable, le escuchaban atentamente, se ponían en su lugar para comprenderlo, y tenían facilidad para acercarse.

En la categoría Priorizar al Ser Cuidado, 71,2\% percibieron como buena, y $10 \%$ excelente la priorización de las enfermeras; aquí se evalúa cuando las enfermeras se mostraron respetuosas, lo ponían en primer lugar, lo llamaban por su nombre, respetaron sus decisiones, y respetaron su intimidad, ante cualquier eventualidad.

En la categoría Disponibilidad para la Atención, 66,2\% percibieron buena, y 12,5\% excelente disponibilidad para la atención de enfermería; es decir el seguimiento frecuente, la dedicación de tiempo, administración del tratamiento ordenado a tiempo, respuesta con rapidez al llamado, identificación de las necesidades, y la importancia del estado de ánimo de los pacientes. 
Tabla 2. Resultados del PCHE por categorías

\begin{tabular}{|c|c|c|c|c|c|}
\hline $\begin{array}{l}\text { PCHE por } \\
\text { categorias }\end{array}$ & $\begin{array}{l}\text { Exce- } \\
\text { Tente }\end{array}$ & Bueno & $\mathbf{R} \boldsymbol{E}$ & Begular & Malo \\
\hline $\begin{array}{l}\text { Sentimientos de los } \\
\text { adultosmayores }\end{array}$ & $38,8 \%$ & $43,8 \%$ & $16,3 \%$ & $1,3 \%$ & \\
\hline $\begin{array}{l}\text { Características de la } \\
\text { enfermera }\end{array}$ & $7,5 \%$ & $65 \%$ & $27,5 \%$ & $0 \%$ & \\
\hline $\begin{array}{l}\text { Del hacer de la } \\
\text { enfermera }\end{array}$ & $2,5 \%$ & $76,3 \%$ & $20 \%$ & $1,3 \%$ & \\
\hline Proactividad & $60 \%$ & $26,3 \%$ & $11,3 \%$ & $2,5 \%$ & \\
\hline Apoyoemocional & $58,8 \%$ & $27,5 \%$ & $13,8 \%$ & $0 \%$ & \\
\hline Apoyofísico & $51,3 \%$ & $32,5 \%$ & $16,3 \%$ & $0 \%$ & \\
\hline Empatía & $3,8 \%$ & $73,8 \%$ & $22,5 \%$ & $0 \%$ & \\
\hline $\begin{array}{l}\text { Priorizar al ser } \\
\text { cuidado }\end{array}$ & $10 \%$ & $71,3 \%$ & $18,8 \%$ & $0 \%$ & \\
\hline $\begin{array}{l}\text { Disponibilidad para } \\
\text { la atención }\end{array}$ & $2,5 \%$ & $\%$ & & $3 \%$ & $0 \%$ \\
\hline
\end{tabular}

De acuerdo a los resultados mostrados en la tabla 3 , en forma global, $68,7 \%$ de los adultos mayores entrevistados, se mostraron

satisfechos con el cuidado de enfermería recibido, $21,3 \%$ indicaron estar poco satisfechos, y $10 \%$ estuvieron muy satisfechos con el cuidado de enfermería recibido.

Tabla 3. Análisis de los resultados de la satisfacción con el cuidado recibido

\begin{tabular}{lc}
\hline $\begin{array}{c}\text { Satisfacción con el } \\
\text { cuidado recibido }\end{array}$ & $\%$ \\
\hline Poco Satisfecho & $21,3 \%$ \\
Satisfecho & $68,7 \%$ \\
Muy satisfecho & $10,0 \%$ \\
\hline \multicolumn{1}{c}{ Total } & $100 \%$ \\
\hline Fuente: Encuesta &
\end{tabular}

En la tabla 4, se muestra los resultados de la prueba estadística ji cuadrada, que determina la relación entre el cuidado de enfermería y la satisfacción por el servicio recibido por parte de los adultos mayores. Con lo cual se prueba la hipótesis de investigación.

En el análisis de correlación de las categorías mediante Ji cuadrada, para 95\% de confianza, se ha determinado que existe relación estadística significativa entre el hacer de la enfermería $\left(X^{2}=26,9\right)$, el apoyo emocional $\left(X^{2}\right.$ $=14,1)$, y la empatía $\left(X^{2}=11,0\right)$, con la satisfacción por el servicio de enfermería recibido.

Las percepciones porcentuales de cada pregunta del cuestionario PCHE, destacan valoraciones de excelente en los siguientes ítems: Son amables en el trato $(53,85 \%)$, Le dan estímulos positivos (53,8\%), Demuestran conocimientos y habilidades profesionales, (55\%), Le explican anticipadamente los procedimientos $(51,3 \%)$, Se identifican antes de realizarle los cuidados $(63,8 \%)$, Dan instrucciones sobre cuidado (55\%), Le dan tranquilidad $(53,8 \%)$, Hacen que el paciente se sienta bien físicamente $(51,3 \%)$, Se muestran respetuosos (67,5\%), Lo llaman por su nombre (76,3\%), Administran el tratamiento ordenado a tiempo $(78,8 \%)$.

Tabla 4. Cuidado de enfermería Vs satisfacción con el servicio

\begin{tabular}{|c|c|c|c|c|c|c|c|c|}
\hline \multirow{3}{*}{$\begin{array}{l}\text { CUIDADO } \\
\text { DE } \\
\text { ENFERME } \\
\text { RÍA }\end{array}$} & \multicolumn{6}{|c|}{$\begin{array}{l}\text { SATISFACCIONCON EL SERVICIO } \\
\text { RECIBIDO }\end{array}$} & \multirow{2}{*}{\multicolumn{2}{|c|}{ TOTAL }} \\
\hline & $\begin{array}{r}P \\
\text { SATIS }\end{array}$ & $\begin{array}{l}\mathrm{CO} \\
\text { ECHO }\end{array}$ & SAT & IISFECHO & $\begin{array}{r}M \\
\text { SATIS }\end{array}$ & $\begin{array}{l}\text { IUY } \\
\text { FECHO }\end{array}$ & & \\
\hline & $\mathrm{N}^{\circ}$ & $\%$ & $\mathrm{~N}^{\circ}$ & $N^{\circ}$ & $\%$ & $-N^{\circ}$ & $\%$ & \\
\hline Excelente & 8 & 10,0 & 21 & 26,3 & 8 & 10,0 & 37 & 46,3 \\
\hline Bueno & 9 & 11,3 & 34 & 42,5 & 0 & 0,0 & 43 & 53,8 \\
\hline TOTAL & 17 & 21,3 & 55 & 68,8 & 8 & 10,0 & 80 & 100,0 \\
\hline
\end{tabular}

\section{DISCUSIÓN.}


En cuanto a las características sociodemográficas, se ha encontrado que los adultos mayores objeto del estudio, fueron mayoritariamente hombres $(56,3 \%)$, en edad promedio de 72,47 años ( $D S=9,737$ ), donde predomina el grupo etario de 65 a 69 años, $63,8 \%$ tienen estudios de primaria 0 secundaria, $32,5 \%$ no trabajan, $21,3 \%$ son amas de casa, $17,5 \%$ presentaban neumonía, y $73,8 \%$ tenían regular estado de salud. Estas características difieren de los indicados en otros estudios debido al contexto y la selección de la población realizada por cada investigador.

Los adultos mayores hospitalizados en los servicios de Medicina y Cirugía del Hospital Santa María del Socorro de Ica, percibieron en forma global el comportamiento de cuidado humanizado por enfermería como bueno $(53,7 \%)$, y excelente (46,3\%). Romero, Contreras y Moncada (2013) (5) encontraron $55,4 \%$ de pacientes hospitalizados que siempre percibieron cuidado humanizado de enfermería, que es un resultado similar al nuestro. Por otra parte, González, y Quintero (11), mencionan $73 \%$ de mujeres hospitalizadas, que siempre percibieron el cuidado humanizado de enfermería, este resultado corresponde a un nivel de buen cuidado, relativamente menor a lo encontrado en nuestro estudio. De igual manera, Peroza, 
Querakes, y Silva (13), dan a conocer sobre el Cuidado Humanizado, que $100 \%$ de pacientes recibieron trato con respeto, amabilidad e igualdad, y $85 \%$ afirman satisfacción con la atención. También Rivera y Triana (21) muestran $86,7 \%$ de pacientes hospitalizados que percibieron siempre un cuidado humanizado. A partir de estos resultados podemos corroborar que independientemente del ámbito institucional donde labore la enfermera, en ellas se perciben capacidades para llevar a cabo la praxis. El cuidado humanizado centrado en el paciente, según los estudios revisados, mayoritariamente son percibidos con índices de niveles buenos y excelentes, lo cual parece correcto que así sea, más aun teniendo en cuenta que enfermería trabaja con personas, a quienes hay que salvares la vida o cuidar de su salud según sea el caso.

En cuanto a las categorías de PCHE, los resultados señalan que las categorías Proactividad (60\%), Apoyo Emocional (58,8\%), Apoyo físico (51,3\%) y Sentimientos del Paciente $(38,8 \%)$ tienen altos índices de valoración como excelentes, y las categorías del Hacer de la Enfermera (76,3\%), Empatía (73,8\%), Priorizar al Ser Cuidado (71,3\%), Disponibilidad para la Atención $(66,3 \%)$ y Características de la Enfermera (65\%) tienen altos índices de valoración como buenos. Rivera y Triana (21) mostraron para cuidado humanizado comportamientos en donde se priorizó a la persona $(89,5 \%)$, cualidades de la enfermera $(89,4 \%)$, características de la enfermera $(89,8 \%)$ y sentimientos del paciente $(87,6 \%)$, los comportamientos de cuidado que fueron percibidos en menor medida por el usuario fueron: Empatía (77,7\%), Apoyo Emocional (79,6\%), Proactividad $(80,5 \%)$ y disponibilidad para la atención $(85,4 \%)$, estos hallazgos también muestran altos índices, en algunos casos similares al hallado en el presente estudio.

En el análisis de la satisfacción con el servicio recibido, en forma global se ha determinado, que $68,7 \%$ de los adultos mayores entrevistados, se mostraron satisfechos con el cuidado de enfermería recibido, 21,3\% indicaron estar poco satisfechos, y $10 \%$ estuvieron muy satisfechos con el cuidado de enfermería recibido. Al respecto López Amasifén, y Rodríguez Curi (8), en su estudio, muestran altos niveles de satisfacción sobre los cuidados que brinda la enfermera en el servicio de Medicina, también Molina Cardona (9), encontró $83 \%$ de alto grado de satisfacción con la calidad del cuidado de enfermería; y Cartagena, Cabarcas, Montes, Ortiz (12), determinaron el nivel de satisfacción del paciente hospitalizado con la atención de enfermería, habiendo encontrado $85 \%$ de satisfacción buena, 15\% regular. En otro estudio, Dolores García M. (22), identifico que más del $50 \%$ de los adultos mayores presentaron nivel de satisfacción alto al cuidado proporcionado por el personal de enfermería, y Gloria Zavala Lizaraso (23) encontró mayores frecuencias de satisfacción Media, siendo la dimensión humana la menos valorada.

\section{CONCLUSIONES}

Existe relación estadística significativa entre el cuidado humanizado de enfermería, y la satisfacción por el servicio recibido en los adultos mayores que acudieron al Hospital Santa María del Socorro de Ica. $\left(X^{2}=11,0 p\right.$, 000). En forma global, los adultos mayores percibieron mayoritariamente como bueno $(53,7 \%)$, el cuidado humanizado de enfermería, y en menor proporción mencionaron que fue excelente $(46,3 \%)$. El $68,7 \%$ de los adultos mayores, se mostraron satisfechos con el cuidado de enfermería recibido, 21,3\% indicaron estar poco satisfechos, y $10 \%$ estuvieron muy satisfechos.

\section{RECOMENDACIONES}

Implementar el cuidado humanizado de enfermería, sensibilizando y preparando al personal de enfermería que labora en los diversos servicios.

\section{Correspondencia:}

Dra. Susana Alvarado Alfaro

Correo: susanasa11@hotmail.com 


\section{REFERENCIAS BIBLIOGRÁFICAS}

1. Watson J. Filosofía y teoría de los cuidados humanos. 3 ed. California: Universidad de Colorado. 1999.

2. Health Canadá. Secretaría de Salud de México y Organización Panamericana de la Salud. Guía para el desarrollo de un sistema integral de apoyo para el envejecimiento activo .p 5. Washington, DC. 2002.

3. OMS. Enseñanza de la Enfermería en Salud del Adulto Mayor. Serie Recursos Humanos para la Salud. Washington DC. 2012.

4. INEI. Perfil sociodemográfico del Perú. Lima, Perú. 2007.

5. Romero $M$; Contreras $M$; Pérez $P$; Moncada A; Jiménez Z. Cuidado Humanizado de enfermería en pacientes hospitalizados, en Cartagena Colombia. 2013. Rev Ciencias Biomédicas: 4 (1):60-68.

6. Rojas M; Barajas L. Percepción de la calidad del cuidado de enfermería en el servicio de urgencias, de la Unidad Básica Puente Barco Leones de La Pamplona España. 2012. Rev España Ciencia y Cuidado. 2012; 9 (1): 13 - 23.

7. Tejada K. Factores Sociodemográficos y su relación con la dimensión de la calidad de atención del cuidado de enfermería en el paciente adulto mayor hospitalizado en el servicio de medicina del Hospital Hipólito Unanue de Tacna. Universidad Nacional Jorge Basadre Grohmann de Tacna. Perú. 2012.

8. López A., Rodríguez C. Nivel de satisfacción del paciente Adulto Mayor sobre el cuidado que brinda la enfermera en el servicio de Medicina del Hospital II2 MINSA-TARAPOTO Periodo Junio - Julio 2012 Facultad de Ciencias de la Salud. Universidad Nacional de San Martín. Tarapoto, Perú. 2012.
9. Molina C. Evaluación de la calidad del cuidado de enfermería en un servicio de hospitalización domiciliaria en Bogotá D.C.. Tesis Maestría. Área temática de gerencia en Salud y Enfermería. 2011.

10. Corrales M. Actitudes de las enfermeras y los enfermeros de cuidado directo y la percepción del adulto mayor sobre el Cuidado recibido. Escuela de Enfermeria . Universidad de Carabobo, Venezuela. 2011

11. González B; Quintero M. Percepción del cuidado humanizado en pacientes que ingresan al servicio de hospitalización de urgencias de ginecoobstetricia de una institución de II y III nivel de atención, Tesis Pregrado. Facultad de enfermería. Pontificia Universidad Javeriano. Bogota D.C. 2009.

12. Cabarcas O; Montes C; Ortiz G. Satisfacción del paciente hospitalizado con la atención de enfermería en una institución de salud de la ciudad de Cartagena, Colombia (Internet). Rev. Colombia de enfermería. Colombia. 2009;

1(1): citado el 14 de Abril 2014Disponible en: http://www.encolombia.com/medicina/enfe rmeria/enfermvol120109/satisfacciondelp

13. Peroza K; Querakes F; Silva M. Calidad De Los Cuidados De Enfermería Dirigidos Al Adulto Mayor En La Unidad Gerontológica "Doña María Pereira De Daza”. Barquisimeto-Estado Lara. Universidad Centroccidental Lisandro Alvarado. Programa de Enfermería. Barquisimeto, Venezuela. 2009.

14. Sarter B. Philosophical sources of nursing theory. En Reed PG, Shearer N, Nicoll L. Perspectives of nursing theories. 4 ed. USA 2004. Lippicontt Williams \& Williams; cap. 32

15. Watson J. Caring science as sacred Science. Philadelphia: FA Davis. 2005.

16. Rogers M. An introduction to the theoretical basis of nursing. Philadelphia: Saunders. 1970. 
17. Leininger $\mathbf{M}$. Caring: an essential human need. Detroit: Slack. 1991.

18. Parse R. Man-living-health: A theory of nursing. New York: Wiley. 1981

19. Pender $\mathbf{N}$. Health promotion in nursing practice. New York: Appleton-Century Crofts. 1982.

20. Newman M. Health as expanding consciousness. St. Louis: Mosby. 1986

21. Rivera L; Triana A. Cuidado humanizado de enfermería: visibilizando la teoría y la investigación en la práctica, en la clínica del country. 2007. Enferm.; 10(4):15-21

22. García M. Satisfacción del usuario adulto mayor con la calidad de la atención de enfermería en el hospital general Cunduacán Tabasco. Rev. Mexico Horizonte Sanitario. Calidad y Gestión en Servicios de Salud. 2008; 7(3).
23. Zavala G. Nivel de satisfacción del paciente adulto mayor sobre el cuidado que brinda la enfermera en el servicio de medicina del Hospital Nacional Dos de Mayo en el 2009. Biblioteca Virtual Nacional Mayor de San Marcos. Lima, Perú. 2009.

Recibido: 27/02/2016

Aprobado para Publicación: 27/05/2016 